\title{
Management of Children Referred to ENT with Suspected Obstructive Sleep Apnea Following Overnight Oximetry.
}

\author{
Original Ahmed Yehia Hussein ${ }^{1}$, Bradley Story², Goubran Eskander, Amr Ossama \\ Article Abdelhamid ${ }^{1,4}$, Atef El-kholy \\ ${ }^{1}$ Department of Otorhinolaryngology, Ain Shams University, ${ }^{2}$ Manchester University of \\ Medicine, ${ }^{3}$ St Helens and Knowsley NHS Trust, ${ }^{4}$ Manchester University NHS Foundation \\ Trust
}

\begin{abstract}
Background: Obstructive sleep apnea (OSA) has been associated with increased risk of respiratory complications after adenotonsillectomy in children. Not all such children require overnight admission, and same-day surgery is appropriate for some patients. Selection of those that are suitable for same day discharge relies on accurate identification of OSA severity.

Guidelines about 'Day Care Adenotonsillectomy in presence of Sleep Apnea” provided by Nottingham Children's Hospital, the UK, published in 2014, and applies to those children who had a sleep study performed pre-operatively.

A typical cost-effective practical sleep study entails at-home overnight pulse oximetry.

Aim: The aim of audit study was to see if suspected OSA is appropriately managed at Trafford General Hospital (TGH), the UK, according to the standards provided by Nottingham Children's Hospital, in addition to taking one standard from the Royal College of Pediatrics and Child Health report, on Standards for Services for Children with Disorders of Sleep Physiology, published in 2009.

Study Design: This is a retrospective local audit study focused on children who were referred to ENT at TGH for suspected OSA or who were suspected of OSA during ENT follow-up for another non-OSA reason.

Conclusion: There is good practice in excluding insufficiently analyzed oximetry.

Non-compliance was mostly due to mild OSA being ruled in on an inconclusive study. The listing and referral for adenotonsillectomy is appropriate despite conflicting local practice to refer all children with suspected OSA to a specialized centre.
\end{abstract}

Key Words: Adenotonsillectomy, obstructive sleep apnea, overnight admission, sleep study

Received: 7 September 2019, Accepted: 14 February 2020

Corresponding Author: Hussein, Ahmed Yehia, M.D., Otorhinolaryngology Department, Faculty of Medicine, Ain Shams University, Egypt, Tel.: 447533265273, E-mail: ahmedyz76@gmail.com

ISSN: 2090-0740, July 2020 Vol.21, No.2

\section{INTRODUCTION}

Auditory Sleep disordered breathing (SDB) is the general term for a range of breathing difficulties occurring during sleep, from primary snoring to obstructive sleep apnea (OSA). OSA is the most common type of SDB, described as the repeated cessation of airflow during sleep caused by a partial or complete obstruction and thus preventing air from entering the lungs. The prevalence of OSA in children is approximately $0.7-1.8 \%$ and peaks between ages of 3 and $6^{[1,2,3]}$.

Polysomnography(PSG)requiresattendancemonitoring as an in-patient, overnight at a specialist center. However it costs more, decreases attendance, and may discriminate based on geographical availability ${ }^{[14]}$. Overnight oximetry can record most of the variables polysomingraphy (PSG) can, except electroencephalography (EEG). On the other hand, the benefit of overnight oximetry, is that it can be performed at home using a small portable machine that is lent out by the investigating department rather than in a specialized sleep center as an in-patient basis. Overnight oximetry can, therefore, be reliably used in most settings as a convenient alternative to PSG even if not the gold standard.

Certain underlying syndromes, such as Marfan's syndrome or Down syndrome, as well as some craniofacial abnormalities can predispose to OSA, though they are uncommon to see in practice ${ }^{[5]}$. The majority of children with OSA, however, have an element of adenotonsillar hypertrophy, particularly grade II tonsils or above $\mathrm{e}^{[6,7]}$.

Not all children with adenotonsillar hypertrophy, however, develop OSA, suggesting that the pathophysiology of OSA also has a dependent functional cause - that is a reduced tone of the pharyngeal constrictor muscles - and possibly other additional factors ${ }^{[8]}$. Further 
evidence to this idea is that most apneas and hypo-apneas occur during REM sleep when muscle tone is decreased ${ }^{[9]}$.

The Royal College of Surgeons of England (RCS) in 2009 mentioned that "of children presenting with clinically significant SDB can be effectively managed by adenotonsillectomy"[10].

This audit study can provide an idea of how suspected OSA in children is currently being managed, and whether it is of an appropriate standard in relation to the general consensus for management of OSA in a District General Hospital (DGH).

Table 1: Standards used in this audit

\section{METHODOLOGY:}

This is a retrospective local audit focused on children who were referred to the ENT Department at TGH for suspected OSA or who were suspected of OSA during ENT follow-up for another non-OSA reason. The sample was obtained from an appointment list of children referred to the pediatric center of TGH, where the overnight oximetry machines were distributed and returned.

This audit monitors the following standards in (Table 1), to examine and evaluate the management of OSA in children at TGH. Management of the patients including pre-assessment, ambulatory care and post-operative care was done in line with guidelines provided by Nottingham Children's Hospital, the UK, published in 2018.

\begin{tabular}{|c|c|c|c|}
\hline & Standard & Source & Evidence \\
\hline 1 & $\begin{array}{l}\text { Interpretation of overnight oximetry } \\
\text { is only attempted if the sleep study } \\
\text { lasts for at least six hours }\end{array}$ & $\begin{array}{l}\text { Standards for Services for Children with } \\
\text { Disorders of Sleep Physiology' from the } \\
\text { Royal College of Paediatrics and Child } \\
\text { Health }\end{array}$ & $\begin{array}{l}\text { Recommended best practice based on } \\
\text { clinical experience of working party }\end{array}$ \\
\hline 2 & $\begin{array}{l}\text { Interpretation of overnight oximetry } \\
\text { is appropriate and reliable }\end{array}$ & $\begin{array}{l}\text { Guidelines on 'Day care Adenotonsillectomy } \\
\text { in presence of sleep apnoea' from } \\
\text { Nottingham Children's Hospital suggest a } \\
\text { key to oximetry classification }\end{array}$ & $\begin{array}{l}\text { Key based on evidence by Nixon et al } \\
2004 \text { in Planning Adenotonsillectomy in } \\
\text { Children with Obstructive Sleep Apnea: } \\
\text { The Role of Overnight Oximetry' }\end{array}$ \\
\hline 3 & $\begin{array}{l}\text { Appropriate management and referral } \\
\text { for Adenotonsillectomy following } \\
\text { overnight oximetry }\end{array}$ & $\begin{array}{l}\text { Guidelines on 'Day care adenotonsillectomy } \\
\text { in presence of sleep apnoea' from Nottingham } \\
\text { Children's Hospital set admission criteria } \\
\text { for overnight observation and for day-case } \\
\text { surgery }\end{array}$ & $\begin{array}{l}\text { RCS identified risk factors associated with } \\
\text { post-op respiratory complications that } \\
\text { would be better suited for postoperative } \\
\text { overnight observation in a center with } \\
\text { available paediatric ICU }\end{array}$ \\
\hline
\end{tabular}

Kamani T. and Daniel M. Day care adenotonsillectomy in presence of sleep apnoea. Nottingham Children's Hospital, 2018. https://www.nuh. nhs.uk/download.cfm?ver=9967 (accessed 31 Mar 2019) ${ }^{[15]}$.

Royal College of Paediatrics and Child Health. Standards for services for children with disorders of sleep physiology. Royal College of Paediatric and child health working party on sleep physiology and respiratory control disorders in childhood. British Paediatric Respiratory Society, 2009.http://www.bprs.co.uk/documents/rcpch_sleep_resp_cont_disorders.pdf (accessed 24 Jan2016)

The study was conducted over one month, and the sample comprised of 60 consecutive children listed in date order for their overnight oximetry sleep study appointment.

The sampling started from an arbitrary point chosen as the last child to be seen mid-December 2015 and went back to early-October 2015 before reaching the 60th child at which point the sampling was stopped. The electronic patient record (EPR) was then used to retrospectively review each child in the sample focusing on the ENT consultation notes and overnight oximetry sleep study. Data was recorded, compiled anonymously and analyzed using Microsoft Excel 2013 (Microsoft, Redmond, Washington, USA). The standards used for this is clarified in (Table 1). After all exclusions, 33 children were able to have their overnight oximetry and management decisions analyzed.

Overnight sleep study was done at home. The parents had appropriate induction and training on how to use the equipment. The results were recorded by devices and data collected later. The parents were initially blinded to the results collected, until they have a clinic discussion with their physician on a separate appointment afterwards.

The physicians in the ENT clinic evaluated the children clinically for symptoms of OSA, which included snoring, mouth breathing, cessation of breathing during sleep, poor quality of sleep and daytime hyper-somnolence. Physicians were Otolaryngology consultants with sufficient experience in evaluating children's sleep disorders. They evaluated the correlation between the results of overnight oximetry and the physicians' own clinical judgment -depending on symptoms and clinical examination- on the diagnosis and management of OSA in those children. They were not blind to the results of the overnight oximetry. The methodology and sampling are detailed in (Figure 1). 
Fig. 1: Sampling of cases

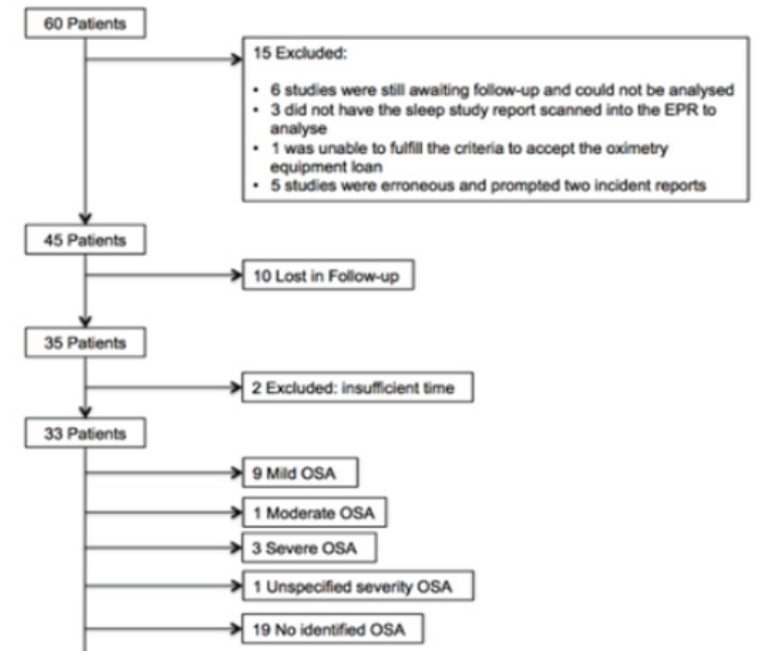

Overnight admission criteria after adenotonsillectomy or performing the operation as day case depends on many variables, our local protocols were used. Pre and post-surgical care local protocols are implemented (see tables 4 to 7 ).

\section{RESULTS}

Analysis of the final sample of 33 children revealed a 1.2:1 male to female ratio with 18 boys and 15 girls. The age of each child in the sample was reported in years except for those under 2 when it was reported in months, and converted to years, as a decimal. The mean average age was $4.53 \pm \mathrm{SD} 0.9$. All further data analysis is mapped to a corresponding audit standard.

Overnight oximetry studies state the duration of the study in minutes as well as the minutes of the study that are actually analyzed. To meet the standards, at least 6 hours of overnight oximetry would need to be analyzed. From the 33 remaining children in the sample, 2 children had less than 6 hours of sleep analyzed and reported as OSA.

To document accurate compliance with this standard, the sample had to include the two children previously excluded due to insufficient study duration, as these demonstrate relevant compliance. Of the 35 children, only 2 were analyzed with duration less than 6 hours (Figure 2). The compliance was $94 \%$.

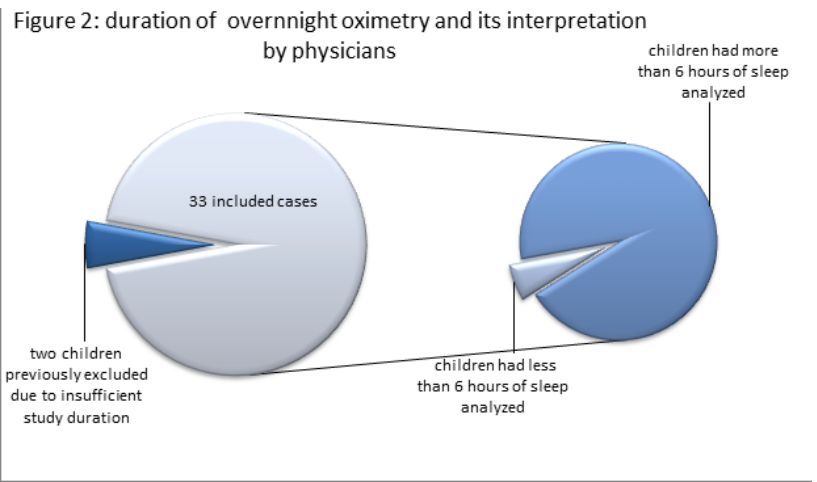

Analysis Using the overnight oximetry guidelines alone predicts that 22 of the 33 children have a normal or inconclusive sleep study (Figure 3). It is expected that the true number of normal studies will be lower and some of these children will instead have mild OSA but an inconclusive study, due to the reported high false negative value of overnight oximetry in the literature. Of the 22 normal sleep studies, the clinician's opinion agreed with 16 (73\%). Of the remaining 6 studies, 5 (24\%) were reported as mild OSA, and $1(5 \%)$ as moderate OSA (Figure 4).

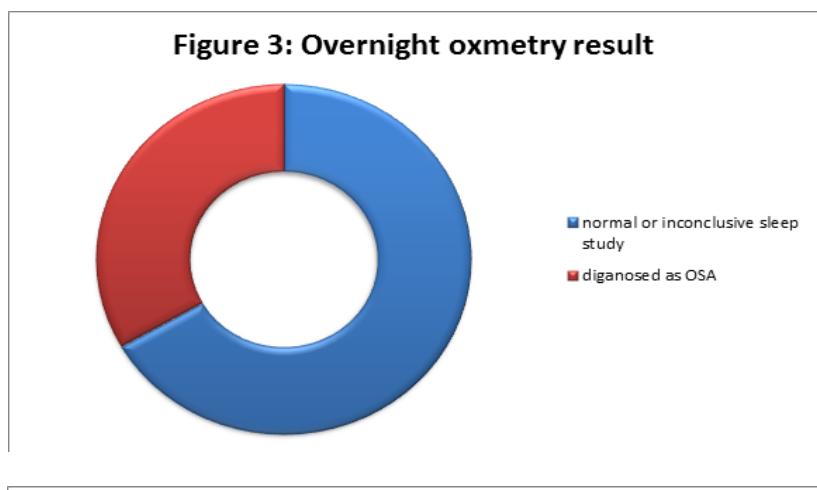

Figure 4: Clinical opinion agreement on overnight oximetry results, when severity was taken into account

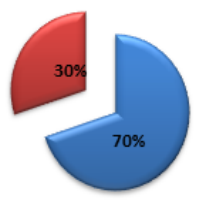

agreed with oximetry results घdisagreed with oximetry results

Of the 11 children in whom OSA was predicted using overnight oximetry guidelines alone, when severity was not taken into account, the clinician's opinion agreed in $8(73 \%)$ sleep studies. Of the remaining $3(27 \%)$ studies, all were reported as having mild OSA by the clinician. If severity was taken into account, the clinician's opinion agreed in 7 (64\%) studies, gave a lower severity compared to the guidelines in $3(27 \%)$, and could not be determined in $1(9 \%)$ study due to no severity being stated.

In total the clinician's opinion agreed on 24 (73\%) sleep studies when severity of OSA was not taken into account and on $23(70 \%)$ sleep studies when severity of OSA was taken into account. In the latter, 1 study was undeterminable because the severity of OSA was not stated. This means the actual percentage of agreement may be as high as $73 \%$. Of the 9 conflicting studies, the clinician's opinion was more severe in $6(67 \%)$ studies and less severe in $3(33 \%)$.

Of the 19 children who had a normal sleep study identified by the clinician, 5 were discharged to the person who made the referral (either GP or pediatrician), 5 were given a review ENT appointment, 5 were listed for adenotonsillectomy at TGH after being deemed beneficial, and 4 were referred for management at The Royal Manchester Children Hospital (RMCH) (Figure 5). 
Of the 5 children listed for adenotonsillectomy at $\mathrm{TGH}$, all $5(100 \%)$ were suitable for DGH adenotonsillectomy.

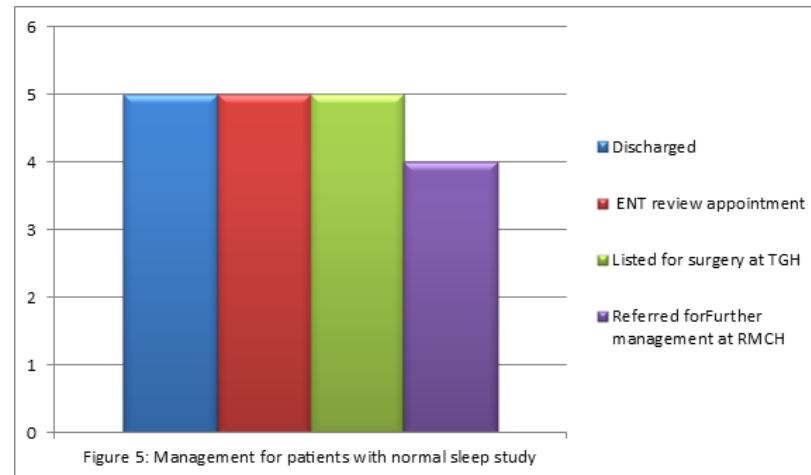

Of the four referred for surgical management and opinion at RMCH, 1 was referred for non-OSA reasons. From the remaining 3, all $3(100 \%)$ were not suitable for DGH adenotonsillectomy due to either age or comorbidities.

Of the 14 children with OSA: 2 children with severe OSA were referred to RMCH for surgical management and opinion, 2 children with moderate OSA were referred to $\mathrm{RMCH}, 9$ children with mild OSA were split between discharge (1), review (3), and referral to RMCH (5), and 1 unknown OSA severity was referred to $\mathrm{RMCH}$.

For the 2 children with severe OSA, the guidelines state that a referral should have been made for both children based on OSA severity alone. For the 2 children with moderate OSA, 1 would have been referred to $\mathrm{RMCH}$ due to a co-morbidity (failure to thrive) and 1 would have fallen into the criteria for day-case DGH adenotonsillectomy; the latter child was referred to RMCH based on TGH practice.

For the 5 children with mild OSA who were referred to $\mathrm{RMCH}, 4$ would have been referred based on the age criterion of the guidelines but 1 would have been eligible for day-case DGH adenotonsillectomy, but still was again referred on TGH practice. For the 1 child with an unknown severity of OSA, comparison with the guidelines cannot be made.

Of the 17 children with or without OSA who were listed or referred for surgical management of OSA with adenotonsillectomy, 15 (88\%) were correctly referred against the standard. The other 2 were referred based on TGH practice of referring all children with OSA to RMCH, though were eligible for day-case DGH adenotonsillectomy and so fall outside of the standard (Figure 6).

Figure 6: Listing or refering children with or without OSA for adenotonsillectomy, comparsion against standard

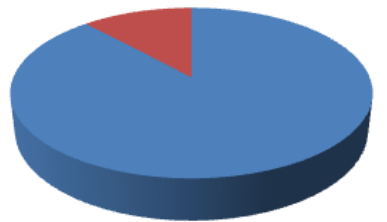

\section{DISCUSSION}

The Symptoms of pediatric OSA slightly differ from adult OSA and include snoring, frequent arousal from sleep, and disordered gas exchange; it is the latter two sequelae that differentiate OSA from primary snoring in children. Moreover, OSA symptoms have fartherreaching complications for children including social problems due to loud snoring, behavioral changes due to inattention or irritability from poor sleep, enuresis, poor growth and failure to thrive, obesity, and increased risk of cardiovascular problems ${ }^{[3]}$.

Obstructive sleep apnea (OSA) in children is usually effectively treated by adenotonsillectomy (T\&A). Studies have shown that a 'normal' child with severe OSA may be more at risk of peri-operative respiratory complications, more sensitive to inhalation anesthetics, more sensitive to opiates, and more likely to have postoperative desaturations ${ }^{[1]}$.

It is now established that those children at risk should be treated with adenotonsillectomy at a specialist pediatric centre with direct and immediate access to pediatric HDU and ICU, and not suitable for district general hospital (DGH). Those risks are clarified in (Table 2) ${ }^{[12]}$.

Table 2: Children at risk from respiratory complications

Children at risk from respiratory complications (unsuitable for DGH adenotonsillectomy according to the Royal College of Surgeons consensus)

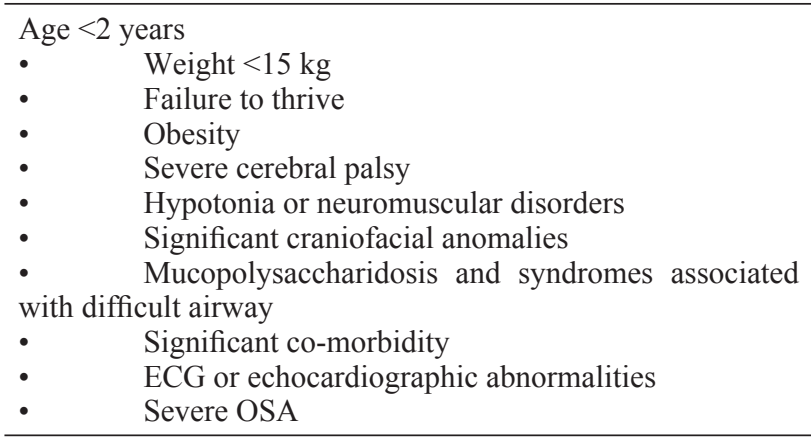

Robb PJ, Bew S, Kubba H, Murphy N, Primhak R, Rollin A-M et al. Tonsillectomyand Adenoidectomy in children with sleep-related breathing disorders: Consensusstatement of a UK Multidisciplinary working party. The Annals of The Royal College of Surgeons of England 2009; 91: 371-373.

Overnight pulse oximetry can be used to estimate the severity of OSA, to shorten the diagnostic and treatment process for those with more severe disease, and to aid clinicians in prioritization of T\&A and planning perioperative care. Nixon et $a l^{[12]}$ in 2004, developed guidance for interpreting overnight oximetry, shown in (Table 3), and thus provided a crude method to assess the severity of $\mathrm{OSA}^{[12]}$. 
Table 3: Guidance for interpreting overnight oximetry studies

\begin{tabular}{cccc}
\hline Guidance for interpreting overnight oximetry studies & \\
OSA Severity & No. of drops per hour & No. of drops per hour & $<5 \%$ \\
Normal / inconclusive & $<90 \%$ & 0 & $\begin{array}{c}\text { No. of drops per hour } \\
<80 \%\end{array}$ \\
Mild OSA & $<3$ & $\leq 3$ & 0 \\
Moderate OSA & $\geq 3$ & $>3$ & 0 \\
Severe OSA & $\geq 3$ & $>3$ & $>3$
\end{tabular}

Nixon GM, Kermack AS, Davis GM, Manoukian JJ, Brown KA, Brouillette RT. Planning Adenotonsillectomy in children with obstructive sleep Apnea: The role of overnight Oximetry. PEDIATRICS 2003; 113: e19-e25

NICE guidelines currently advise "to refer children to a pediatric ENT specialist if they have clinical features of adenotonsillar hypertrophy, symptoms of persistent snoring and features of OSA ${ }^{[4]}$.

It is logical that a significant less sleep would lead to fewer potential desaturations and thus produce an in accurate prediction of OSA severity. The Royal College of Pediatrics and Child Health have since suggested that a minimum of 6 hours sleep be recorded to obtain a reliable overnight oximetry study ${ }^{[13]}$. Studies showed that children with a positive clinical assessment of OSA but negative PSG have significant improvement after T\&A as compared with observation alone, thus validating the clinician's role in diagnosing OSA in children ${ }^{[16]}$.

The results were compared with the standards, aiming to compare practice in relation to the general consensus for management of OSA in a DGH. Different aspects were analyzed, as follows:

1. Interpretation of overnight oximetry is only attempted if the sleep study lasts for at least six hours:

TGH complied with this standard in $94 \%$ of children: either, if the duration of the study fell below this and the child and parent/guardian were called to repeat the study, or, if the duration met or exceeded the requirement; then interpretation was made. The compliance of $94 \%$ represents good compliance and good practice, however, pragmatically it may be considered to be $100 \%$.

2. Interpretation of overnight oximetry is appropriate and reliable

An ENT surgeon may interpret the overnight oximetry sleep study at their discretion in light of the clinical features. The clinician's opinion of whether a child has OSA symptoms was obtained from the clinical consultation notes and compared against what the standard for overnight oximetry interpretation would predict by itself. One drawback for this is that the clinicians were not blind to the studies, which might have resulted in clinical bias.

The study demonstrated the biggest difference of OSA severity suggested by the clinician on the background of a normal or inconclusive study (using the standard). It is surprising how this could be interpreted this way, given that moderate OSA should produce a more conclusive sleep study. Again, this is perhaps reflective of the clinical features not accurately demonstrated in the EPR notes and overnight oximetry having a high false negative value; clinicians account for this by ruling-in mild OSA rather than ruling it out.

This audit verified the proposed standard from guidelines of Nottingham's Children's Hospital, to assess whether interpretation of overnight oximetry was appropriate and reliable or not. Compliance was $70 \%$, which may seem somewhat low, where there was non-compliance, however, was mostly $(15 \%)$ with ruling in mild OSA on the background of a normal or inconclusive study. This is defensive medicine in line with the known high false negative value of overnight oximetry. As noted in the results, only in $1(3 \%)$ case was moderate OSA ruled in on an inconclusive study and in $3(9 \%)$ instances was mild OSA ruled out on the background of predicted mild OSA.

3. Appropriate management and referral for adenotonsillectomy following overnight oximetry

The guidelines from Nottingham Children's Hospital regarding eligibility for day-case surgery (Table 4) only differ from RCS consensus in the minimum age requirement of $\geq 3$ years old compared to $\geq 2$ years old. The guidelines, however, only identify whether a child is suitable for DGH day-case adenotonsillectomy or not based on relevant risk factors. They do not establish whether adenotonsillectomy is suitable; this must be decided using details from the clinical history and examination. 
Table 4: Criteria for Day-case surgery criteria following adenotonsillectomy for OSA

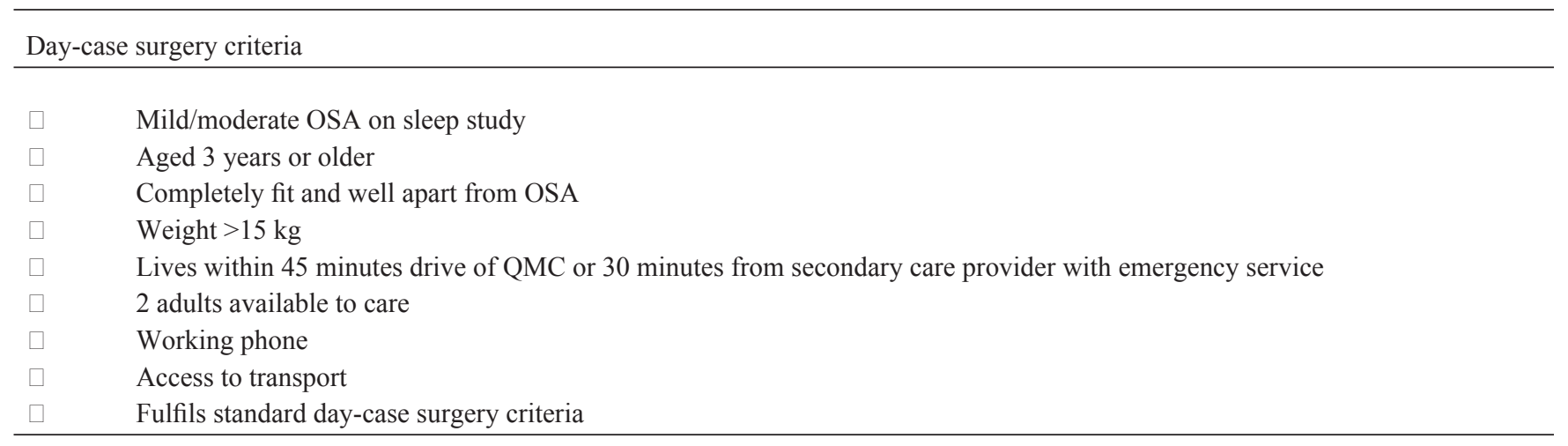

Kamani T. and Daniel M. Day care adenotonsillectomy in presence of sleep apnoea. International journal of pediatric otorhinolaryngology $79(12) \cdot$ October 2015

This audit looked at the appropriate management and referral for adenotonsillectomy following overnight oximetry, assuming adenotonsillectomy was decided to be clinically beneficial.

A comparison was made between how the clinician managed the child and what the guidelines suggest taking into account the relevant risk factors.

Compliance with this standard was $88 \%$ (were correctly referred against the standard). If this consensus (that is, all children suspected of OSA to be referred to $\mathrm{RMCH}$ for postoperative overnight observation) was used as the standard, then, compliance would be $100 \%$.

The limitations of our study are its retrospective nature and the relatively small sample size. The physicians were not blinded to the oximetry results, and their final clinical judgment for management included the oximetry results. This was an advantage for the patients' clinical evaluation, but might have resulted in bias.

\section{CONCLUSION}

Application On the basis of this audit, an agreed action plan was established entailing the following tasks:

Continue to screen for the duration of oximetry analyzed to exclude and repeat invalid studies through Clinicians interpreting sleep studies

Distribution of agreed guidelines based on those used in this audit may be beneficial and provide reliability to the ambiguity of overnight oximetry, and managers at TGH were also informed to help doing that.

Clinicians should continue to refer all children suspected of OSA, or those without OSA but with risk factors of postoperative respiratory complications, to $\mathrm{RMCH}$.

A discussion to reassess if children with mild and moderate OSA without risk factors could all have adenotonsillectomy performed as a day-case at TGH, whether they could be performed if first on morning list, or if a policy to refer all children with OSA should continue to be implemented Clinicians and managers for 12 months.

\section{CONFLICT OF INTEREST}

There are no conflicts of interest.

\section{ETHICAL CONSIDERATIONS}

No ethical approval was required for this study, according to the National Health Service Health Research Authority decision-making tool. Local audit office approval was obtained. All data collected were anonymised.

\section{REFERENCES}

1. Powell S, Kubba H, O'Brien C, Tremlett M. Paediatric obstructive sleep apnoea.BMJ 2010; 340: 1918.

2. Newson L, Rull G. Obstructive Sleep Apnoea in Children. Patient. (2015.http://patient.info/doctor/ obstructive-sleep-apnoea-syndrome-in-children (accessed 24Jan2016).

3. American Academy of Otolaryngology - Head and Neck Surgery. Pediatric sleepdisordered breathing/obstructive sleep Apnea. (2015.http:// www.entnet.org/content/pediatric-sleep-disorderedbreathingobstructive-sleep-apnea (accessed 19 Jan 2016).

4. National Institute for Health and Care Excellence. Obstructive sleep apnoea syndrome. Clinical 
Knowledge Summaries. (2015.http://cks.nice.org. uk/obstructivesleep-apnoea-syndrome\#! scenario (accessed 19 Jan 2016).

5. Fitzgerald NM, Fitzgerald DA. Managing snoring and obstructive sleep apnoea in childhood. Journal of Paediatrics and Child Health 2013; 49: 800-806.

6. Li AM, Wong E, Kew J, Hui S, Fok T. Use of tonsil size in the evaluation of obstructive sleep apnoea. Archives of Disease in Childhood 2002; 87: 156-159.

7. Cahali MB, Soares CF de P, Dantas DA da S, Formigoni GGS. Tonsil volume, tonsilgrade and obstructive sleep apnea: Is there any meaningful correlation? Clinics (SauPaulo) 2011; 66: 1347-1352.

8. Marcus CL. Pathophysiology of childhood obstructive sleep apnea: Currentconcepts. Respiration Physiology 2000; 119: 143-154.

9. Brouillette RT, Morielli A, Leimanis A, Waters KA, Luciano R, Ducharme FM.Nocturnal pulse Oximetry as an abbreviated testing modality for pediatric obstructivesleep Apnea (OSA). Pediatric Research 1999; 45: 44A-44A.

10. Robb PJ, Bew S, Kubba H, Murphy N, Primhak R, Rollin A-M et al. Tonsillectomy and Adenoidectomy in children with sleep-related breathing disorders: Consensus statement of a UK Multidisciplinary working party. The Annals ofThe Royal Collegeof Surgeons of England 2009; 91: 371-373.
11. Min Y. Treatment of obstructive sleep apnea in children. Korean Journal of Pediatrics 2010; 53: 872879 .

12. Nixon GM, Kermack AS, Davis GM, Manoukian JJ, Brown KA, BrouilletteRT. Planning Adenotonsillectomy in children with obstructive sleep Apnea: The role of overnight Oximetry. PEDIATRICS 2003; 113: e19-e25.

13. Royal College of Paediatrics and Child Health. Standards for services for childrenwith disorders of sleep physiology. Royal College of Paediatric and child health working party on sleep physiology and respiratory control disorders in childhood. British Paediatric Respiratory Society, 2009.http://www.bprs. co.uk/documents/rcpch_sleep_resp_cont_disorders. pdf (accessed 24 Jan2016).

14. Scott A, Baltzan M, Wolkove N. Examination of pulse oximetry tracings to detect obstructive sleep apnea in patients with advanced chronic obstructive pulmonary disease. Canadian Respiratory Journal 2014; 21: 171175.

15. Kamani T. and Daniel M. Day care adenotonsillectomy in presence of sleep apnoea. NottinghamChildren's Hospital, 2018. https://www.nuh.nhs.uk/download. cfm?ver=9967 (accessed 31 Mar 2019).

16. Goldstein NA1, Pugazhendhi V, Rao SM, Weedon J et al. Clinical assessment of pediatric obstructive sleep apnea.mPediatrics. 2004 Jul;114(1):33-43. 